\title{
Cos \\ Topological semimetal and Fermi-arc surface states in the electronic structure of pyrochlore iridates
}

\author{
Xiangang Wan, ${ }^{1}$ Ari M. Turner, ${ }^{2}$ Ashvin Vishwanath, ${ }^{2,3}$ and Sergey Y. Savrasov ${ }^{1,4}$ \\ ${ }^{1}$ National Laboratory of Solid State Microstructures and Department of Physics, Nanjing University, Nanjing 210093, China \\ ${ }^{2}$ Department of Physics, University of California, Berkeley, California 94720, USA \\ ${ }^{3}$ Materials Sciences Division, Lawrence Berkeley National Laboratory, Berkeley, California 94720, USA \\ ${ }^{4}$ Department of Physics, University of California, Davis, One Shields Avenue, Davis, California 95616, USA
}

(Received 23 February 2011; published 2 May 2011)

\begin{abstract}
We investigate novel phases that emerge from the interplay of electron correlations and strong spin-orbit interactions. We focus on describing the topological semimetal, a three-dimensional phase of a magnetic solid, and argue that it may be realized in a class of pyrochlore iridates (such as $\mathrm{Y}_{2} \mathrm{Ir}_{2} \mathrm{O}_{7}$ ) based on calculations using the LDA $+U$ method. This state is a three-dimensional analog of graphene with linearly dispersing excitations and provides a condensed-matter realization of Weyl fermions that obeys a two-component Dirac equation. It also exhibits remarkable topological properties manifested by surface states in the form of Fermi arcs, which are impossible to realize in purely two-dimensional band structures. For intermediate correlation strengths, we find this to be the ground state of the pyrochlore iridates, coexisting with noncollinear magnetic order. A narrow window of magnetic "axion" insulator may also be present. An applied magnetic field is found to induce a metallic ground state.
\end{abstract}

DOI: 10.1103/PhysRevB.83.205101

PACS number(s): 71.27.+a, 03.65.Vf

Previously, some of the most striking phenomena in solids, such as high-temperature superconductivity ${ }^{1}$ and colossal magnetoresistance, ${ }^{2}$ were found in transition-metal systems involving $3 d$ orbitals with strong electron correlations. Now it has been realized that in $4 d$ and $5 d$ systems, whose orbitals are spatially more extended, a regime of intermediate correlation appears. Moreover, they display significant spin-orbit coupling, which modifies their electronic structure as recently verified in $\mathrm{Sr}_{2} \mathrm{IrO}_{4}$ (Ref. 3). This is a largely unexplored domain, but already tantalizing new phenomena have been glimpsed. For example, in the $5 d$ iridium-based magnetic insulator, $\mathrm{Na}_{4} \mathrm{Ir}_{3} \mathrm{O}_{8}$ (Ref. 4), a disordered ground state persists down to the lowest measured temperatures, making it a prime candidate for a quantum spin liquid. ${ }^{5}$

It is known that strong spin-orbit interactions can lead to a novel phase of matter, the topological insulator. ${ }^{6}$ However, the bismuth-based experimental realizations uncovered so far have weak electron correlations. Recently, it was pointed out that the iridium oxides (iridates) are promising candidates to realize topological insulators ${ }^{7}$ and that iridium-based pyrochlores in particular $^{8}$ provide a unique opportunity to study the interplay of Coulomb interactions, spin-orbit coupling, and the band topology of solids.

The main focus of our work is the pyrochlore iridates, which have the general formula $A_{2} \mathrm{Ir}_{2} \mathrm{O}_{7}$, where $A=$ yttrium or a lanthanide element. Experiments on these materials indicate magnetic order. ${ }^{9,10}$ Thus, the possible phases have not been treated in the theory of topological insulators, which assumes time-reversal symmetry. A rather different, but also unusual phase, the topological semimetal is predicted by our LSDA $+U+$ SO (where LSDA stands for local-spin-density approximation and SO stands for spin orbit) calculations in a range of parameters appropriate to the iridates. This phase has linearly dispersing excitations $a t$ the chemical potential, analogous to graphene, ${ }^{11}$ but occurs inside a fully threedimensional magnetic solid. The small density of states leads to a vanishing conductivity at low temperatures. Each mode in this metal is described by a two-component wave-function (described by the "Weyl equation," the two-component analog of the Dirac equation), describing a point where two bands touch. The Weyl equation is used in particle physics to describe the chiral and massless behavior of neutrinos (in limits where their small mass can be neglected). Hence, we also call it the "Weyl semimetal."

Weyl fermions can be assigned a chirality; that is, they are either left or right handed. These modes cannot be gapped unless they mix with a fermion of opposite handedness, which is located at a different point in the Brillouin zone. Thus the gaplessness of Weyl fermions is absolute provided momentum is conserved $;{ }^{12}$ it does not require any fine-tuning or symmetry. These modes are most robust in systems with magnetic order. They do not exist at all if both time reversal and inversion symmetry are present, for example, in bismuth. There, in contrast, Dirac fermions with four-component wave functions appear, which are typically gapped.

A key property of the Weyl semimetal phase studied in this work is its unusual surface states, reminiscent of topological insulators. Since the bulk fermi surface only consists of a set of momentum points, surface states can be defined for nearly every surface momentum and take the shape of "Fermi arcs" in the surface Brillouin zone that stretch between Weyl points.

The "axion insulator" phase can emerge when the Weyl points annihilate in pairs as the correlations are reduced. This phase shows a topological magnetoelectric effect, ${ }^{13}$ captured by the magnetoelectric parameter $\theta=\pi$, whose value is protected by the inversion symmetry, which is respected in our system. The name axion insulator refers to the analogy with the axion vacuum in particle physics. ${ }^{14}$

In the pyrochlore iridates both the $A$ and the Ir atoms are located on a network of corner-sharing tetrahedra. ${ }^{15,16}$ Pioneering experiments ${ }^{17}$ revealed an evolution of groundstate properties with increasing radius of the $A$ ion, which is believed to tune electron correlations. While $A=\operatorname{Pr}$ is metallic, $A=\mathrm{Y}$ is an insulator at low temperatures. Subsequently, 
it was shown that the insulating ground states evolve from a high-temperature metallic phase via a magnetic transition. ${ }^{9,10}$ The magnetism was shown to arise from the Ir sites, since it also occurs in $A=\mathrm{Y}, \mathrm{Lu}$, where the $A$ sites are nonmagnetic. While its precise nature remains unknown, ferromagnetic ordering is considered unlikely, since magnetic hysteresis is not observed.

We show that electronic structure calculations can naturally account for this evolution and point to a novel ground state. First, we find that magnetic moments order on the Ir sites in a noncollinear pattern with moment on a tetrahedron pointing all in or all out from the center. This structure retains inversion symmetry, a fact that greatly aids the electronic structure analysis. While the magnetic pattern remains fixed, the electronic properties evolve with correlation strength. For weak correlations, or in the absence of magnetic order, a metal is obtained, in contrast to the interesting topological insulator scenario of Ref. 8. With strong correlations we find a Mott insulator with all-in/all-out magnetic order. However, for the case of intermediate correlations, relevant to $\mathrm{Y}_{2} \mathrm{Ir}_{2} \mathrm{O}_{7}$, the electronic ground state is found to be a Weyl semimetal, with linearly dispersing Dirac nodes at the chemical potential and other properties described above.

We also mention the possibility of an exotic insulating phase emerging when the Weyl points annihilate in pairs as the correlations are reduced; we call it the $\theta=\pi$ axion insulator. Although our LSDA $+U+\mathrm{SO}$ calculations find that a metallic phase intervenes before this possibility is realized, we note that local-density approximation (LDA) systematically underestimates gaps, so this scenario could well occur in reality. Finally, we mention that modest magnetic fields could induce a reorientation of the magnetic moments, leading to a metallic phase. Previous studies include Ref. 18, an ab initio study which considered ferromagnetism. In Ref. 19, the tight-binding model of Ref. 8 was extended to include tetragonal crystal fields, but in the absence of magnetism. The topological Dirac metal and axion insulator discussed here do not appear in those works, largely due to the difference of magnetic order from our study.

We begin by giving a brief overview of the theoretical ideas that will be invoked in this work, before turning to our LSDA $+U$ calculations of magnetic and electronic structure of the pyrochlore iridates. We then discuss the special surface states that arise in the Weyl semimetal phase and close with a comparison to existing experiments and conclusions. Our results are summarized in the phase diagram Fig. 1.

\section{WEYL SEMIMETALS AND INVERSION-SYMMETRIC INSULATORS}

Weyl points are points where the valence band and conduction band touch. The excitations near each Weyl point $\mathbf{k}_{0}$ are described by an effective Hamiltonian:

$$
H_{D}=E_{0} \mathbb{1}+\mathbf{v}_{0} \cdot \mathbf{q} \mathbb{1}+\sum_{i=1}^{3} \mathbf{v}_{i} \cdot \mathbf{q} \sigma_{i}
$$

Energy is measured from the chemical potential, $\mathbf{q}=\mathbf{k}-\mathbf{k}_{0}$ and $\left(\mathbb{1}, \sigma_{i}\right)$ are the identity matrix and three Pauli matrices, respectively. This Hamiltonian is obtained by expanding the

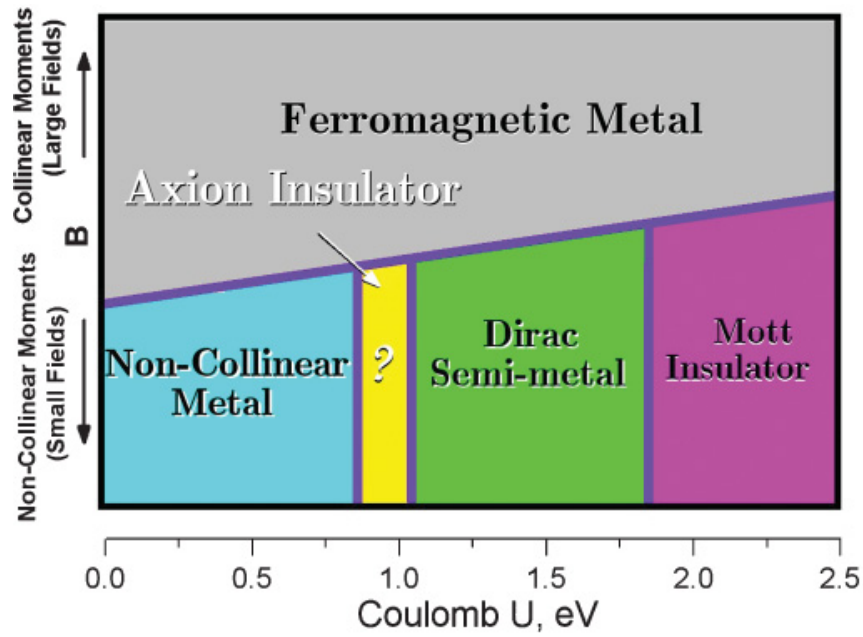

FIG. 1. (Color online) Sketch of the predicted phase diagram for pyrochlore iridiates. The horizontal axis corresponds to the increasing interaction among Ir $5 d$ electrons while the vertical axis corresponds to external magnetic field, which can trigger a transition out of the noncollinear "all-in/all-out" ground state, which has several electronic phases.

full Hamiltonian to linear order. No assumptions are needed beyond the requirement that the two eigenvalues become degenerate at $k_{0}$. The velocity vectors $\mathbf{v}_{i}$ are generically nonvanishing and linearly independent. The energy dispersion is conelike, $\Delta E=\mathbf{v}_{0} \cdot \mathbf{q} \pm \sqrt{\sum_{i=1}^{3}\left(\mathbf{v}_{i} \cdot \mathbf{q}\right)^{2}}$. One can assign a chirality (or chiral charge) $c= \pm 1$ to the fermions defined as $c=\operatorname{sgn}\left(\mathbf{v}_{1} \cdot \mathbf{v}_{2} \times \mathbf{v}_{3}\right)$. Note that, since the $2 \times 2$ Pauli matrices appear, our Weyl particles are two-component fermions. In contrast to regular four component Dirac fermions, it is not possible to introduce a mass gap. The only way for these modes to disappear is if they meet with another two-component Weyl fermion in the Brillouin zone, but with opposite chiral charge. Thus, they are topological objects. By inversion symmetry, the band touchings come in pairs, at $\mathbf{k}_{\mathbf{0}}$ and $-\mathbf{k}_{\mathbf{0}}$, and these have opposite chiralities (since the velocity vectors are reversed).

This semimetallic behavior would not occur (generically) in a system without magnetic order. In materials such as bismuth, with both time reversal and inversion symmetry, Dirac fermions always contain both left- and right-handed components and are thus typically gapped. ${ }^{20}$

When the compound has stoichiometric composition, and all the Weyl points are related by symmetry, the Fermi energy can generically line up with the energy of the touching points. Under these circumstances, the density of states is equal to zero and the behavior of the Weyl fermions controls the low-temperature physics of the solid. For example, the ac conductivity should have a particular frequency dependence, and novel types of surface states should occur, as discussed below. Because of the symmetry relating the Weyl points, their energies $E_{0}$ must coincide. Then, the Fermi energy is fixed at the touching points because of the Kohn-Luttinger theorem: At stoichiometry, there are an integer number of electrons per unit cell. Hence, the Kohn-Luttinger theorem implies that the volume of particlelike minus holelike Fermi surfaces must be a multiple of the volume of the Brillouin 
zone. If the Fermi surface were slightly above (below) $E_{0}$, then the Fermi surfaces would all be particle- (hole)-like, and the Kohn-Luttinger theorem would not be satisfied.

Inversion symmetry provides useful information about the materials we study. Inversion about the origin is given by $\mathbf{r} \rightarrow-\mathbf{r}$; this is a symmetry of many crystals and, in particular, of the ones we focus on here. Inversion strongly constrains many physical properties; in particular, it leads to a quantized magnetoelectric susceptibility (see Sec. III) and can help pin the Fermi energy exactly at Weyl points. Finally, because of inversion symmetry, a few states in the band structure determine many basic properties of a solid. The symmetry relates crystal momenta $\pm \mathbf{k}$. At special momenta, called TRIMs (time-reversal invariant momenta), which are invariant under inversion, the states can be labeled by parity eigenvalues $\xi= \pm 1$. These eigenvalues can be used to identify phase transitions as parameters of the system are changed. Whenever the set of eigenvalues associated with occupied states changes, there must be a phase transition. The highest energy level at a TRIM in the valence band can cross through the lowest energy level in the conduction band if their parities are different, and this changes the set of $\xi$ 's at that TRIM. Hence, the gap passes through 0 . In fact, more is true: The compound cannot be insulating on both sides of the transition. ${ }^{21} \mathrm{~A}$ pair (or an odd number of pairs) of Weyl points emerge from or are absorbed into the TRIM after the parities change (see the Appendix for a derivation in the context of the iridates).

We now turn to results for a particular family of materials, the pyrochlore iridates, where we argue that these general ideas are realized.

\section{LDA CALCULATION AND MAGNETIC ORDER}

We focus here on our numerical ab initio studies of the compound $\mathrm{Y}_{2} \mathrm{Ir}_{2} \mathrm{O}_{7}$. We have also done calculations on iridates with rare earth atoms $(\mathrm{Pr}, \mathrm{Nd}, \mathrm{SmE} u)$ at the $A$ site. Changing the $A$ site has essentially no effect except for changing the appropriate value of $U$, the interaction parameter.

The strength of the SO coupling is large for Ir $5 d$ electrons and leads to insulating behavior in $\mathrm{Sr}_{2} \mathrm{IrO}_{4}$ (Ref. 3); we need to take it into account in order to understand the magnetic and electronic properties.
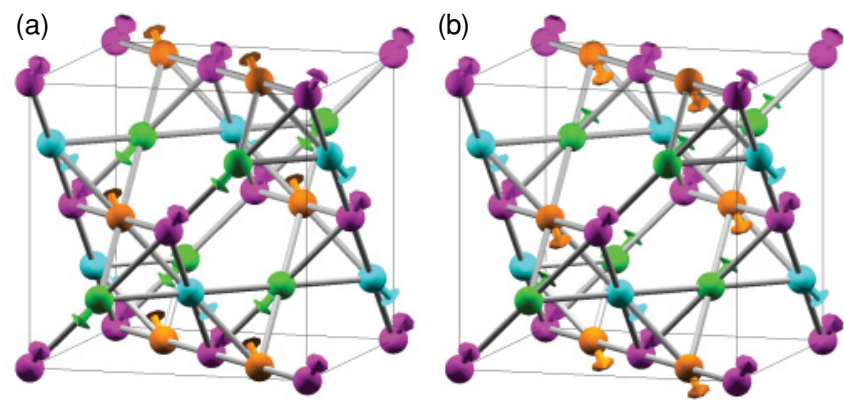

FIG. 2. (Color online) The pyrochlore crystal structure showing the Ir corner sharing tetrahedral network and two of the possible magnetic configurations. (a) The configuration that is predicted to occur for iridates, with all-in/all-out magnetic order. (b) An alternative, the 2-in/2-out configuration.
The system contains four iridium atoms inside each unit cell forming a tetrahedral network as shown in Fig. 2. We perform our electronic structure calculations based on local spin density approximation to density functional theory (DFT) with the full-potential, all-electron, linear-muffin-tin-orbital (LMTO) method. ${ }^{22}$ We use a LSDA $+U$ scheme $^{23}$ to take into account the electron-electron interaction between $\operatorname{Ir} 5 d$ electrons and vary parameter $U$ between 0 and $3 \mathrm{eV}$ for $\operatorname{Ir} 5 d$ electrons to see what effects the on-site Coulomb repulsion would bring to the electronic structure of iridates. In general, we expect that $U$ can be somewhere between 1 and $2 \mathrm{eV}$ for the extended $5 d$ states. We use a $24 \times 24 \times 24 k$ mesh to perform Brillouin-zone integration and switch off symmetry operations in order to minimize possible numerical errors in studies of various (non-)collinear configurations. We use experimental lattice parameters ${ }^{9}$ in all setups.

With only the Heisenberg interaction (and no SO coupling), spins in the pyrochlore configuration would be geometrically frustrated, so we search for the proper magnetic configuration by starting from a large number (12) of different states. The collinear ones were (i) ferromagnetic, with moment along (100), (111), (110), or (120) directions, and (ii) antiferromagnetic with two moments in a tetrahedron along and the other two pointed oppositely to the directions above; noncollinear structures were (iii) the all-in/all-out pattern (all moments point to or away from the centers of the tetrahedron), (iv) "2-in/2-out" (two moments in a tetrahedron point to the center of this tetrahedron, while the other two moments point away from the center, that is, the spin-ice ${ }^{24}$ configuration), and (v) "3-in/1-out" magnetic structures. We show several magnetic configurations in Fig. 2.

We find that the all-in/all-out configuration is the ground state. In contrast to other magnetic configurations, during the self-consistent iterations the spins in the all-in/all-out state retain their initial input direction; indicating a local energy minimum. This state is consistent with the absence of magnetic hysteresis in experiments. ${ }^{9}$ Since all states considered were translationally invariant $q=0$ states, the possibility of complex larger $q$ states cannot be ruled out.

This particular order is, in fact, consistent with the predictions of Ref. 25 for half-integer spins with SO coupling on a pyrochlore lattice, the strongly insulating limit of the present problem. The Dzyaloshinsky-Moriya interaction (generated by the SO coupling) removes the frustration of the Heisenberg antiferromagnet.

Symmetry dictates the form of Dzyaloshinsky-Moriya interactions except for the sign, which leads to two cases, direct and indirect Dzyaloshinsky-Moriya. The all-in/all-out state is the unique ground state for the former while the indirect Dzyaloshinsky-Moriya ground state is a coplanar state with the four spins being either antiparallel or orthogonal. ${ }^{25}$ In our case, the indirect Dzyaloshinsky-Moriya pattern also has higher energy than the all-in/all-out state. This correspondence with the magnetic structure in the strong correlation limit points to the simplest magnetic phase diagram of one magnetic structure spanning a range of correlation strengths.

The next-lowest energy configuration is the ferromagnetic state. Interestingly, the rotation of magnetization does not cost much energy despite strong SO interactions. The (111) direction is found to be the lowest ferromagnetic state, but the 
TABLE I. The spin $\langle S\rangle$ and orbital $\langle O\rangle$ moment (in $\mu_{B}$ ) and the total energy $E_{\text {tot }}$ per unit cell (in meV) for several selected magnetic configurations of $\mathrm{Y}_{2} \mathrm{Ir}_{2} \mathrm{O}_{7}$ as calculated using the LSDA $+U+\mathrm{SO}$ method with $U=1.5 \mathrm{eV}$. ( $E_{\text {tot }}$ is defined relative to the ground state.) The IDM (indirect Dzyaloshinsky-Moriya) is a coplanar configuration predicted for one sign of D-M interactions in Ref. 25.

\begin{tabular}{lccccc}
\hline \hline Configuration: & $(001)$ & $(111)$ & 2-in/2-out & IDM & all-in/all-out \\
\hline$\langle\mathrm{S}\rangle$ & 0.08 & 0.10 & 0.09 & 0.06 & 0.13 \\
$\langle\mathrm{O}\rangle$ & 0.09 & 0.10 & 0.07 & 0.06 & 0.12 \\
$E_{\text {tot }}(\mathrm{meV})$ & 5.47 & 1.30 & 3.02 & 2.90 & 0.00 \\
\hline \hline
\end{tabular}

energy difference between this and the highest energy (001) state is just about $4.17 \mathrm{meV}$ per unit cell. Also, all of them produce a considerable net magnetic moment in contrast to the experiment. ${ }^{9,17}$ Our findings are summarized in Table I for a typical value of $U=1.5 \mathrm{eV}$, and similar results are found for other values of $U$ in the range from 0 to $3 \mathrm{eV}$. Since the energy difference between the ground state and the other magnetic orderings is small, modest magnetic fields may induce a transition into the ferromagnetic state.

Similarly, we note that the honeycomb-lattice Ir compound- $\mathrm{Na}_{2} \mathrm{IrO}_{3}$-initially proposed as a two-dimensional quantum spin Hall insulator, ${ }^{7}$ was predicted by LDA + U to be a magnetically ordered Mott phase, ${ }^{26}$ for which some recent experimental support has emerged. ${ }^{27}$

\section{ELECTRONIC PHASES}

We now discuss electronic properties of iridates that emerge from our LSDA $+U+$ SO calculations. A variety of phases ranging from normal metal at small $U$ to Weyl semimetallic at intermediate $U \sim 1.5 \mathrm{eV}$ and Mott insulating phase at $U$ above $2 \mathrm{eV}$ with noncollinear magnetic all-in/all-out ordering are predicted. Since pressure or chemical substitution may alter the screening and the electronic bandwidth resulting in changes in $U$, we expect that these phases can be observed experimentally in iridates.

The basic features of the electronic structure can be understood by noting that each $\mathrm{Ir}^{4+}$ is coordinated by six $\mathrm{O}^{2-}$ forming approximately an octahedron. The Ir $5 d$ orbitals split into an $e_{g}$ doublet and $t_{2 g}$ triplet. Due to the extended nature of the Ir $5 d$ orbital, the crystal-field splitting between $t_{2 g}$ and $e_{g}$ is large with the $e_{g}$ band to be $2 \mathrm{eV}$ higher than the Fermi level. The bands near the Fermi level are mainly contributed by $\operatorname{Ir} t_{2 g}$ with some mixing with $\mathrm{O} 2 p$ states. SO coupling has a considerable effect on these $t_{2 g}$ states: It lifts their degeneracy and produces a quadruplet with $J_{\text {eff }}=3 / 2$ and a higher-energy Kramers doublet with $J_{\text {eff }}=1 / 2$ (Ref. 3). The five $d$ electrons of $\mathrm{Ir}^{4+}$ fill the quadruplet, and half fill the remaining $J_{\text {eff }}=1 / 2$ orbital. The latter can also be viewed as a $\Gamma_{7}$ doublet, from the strong SO limit. Thus, given the four Ir atoms in the unit cell, we expect eight energy bands near the Fermi energy, which are at half filling.

The precise behavior of these electronic states depends on the magnetic configuration. Our band structure calculations for collinear alignments of moments show metallic bands, a result that disagrees with the insulating behavior found experimentally. Increasing $U$ cannot solve this problem, and

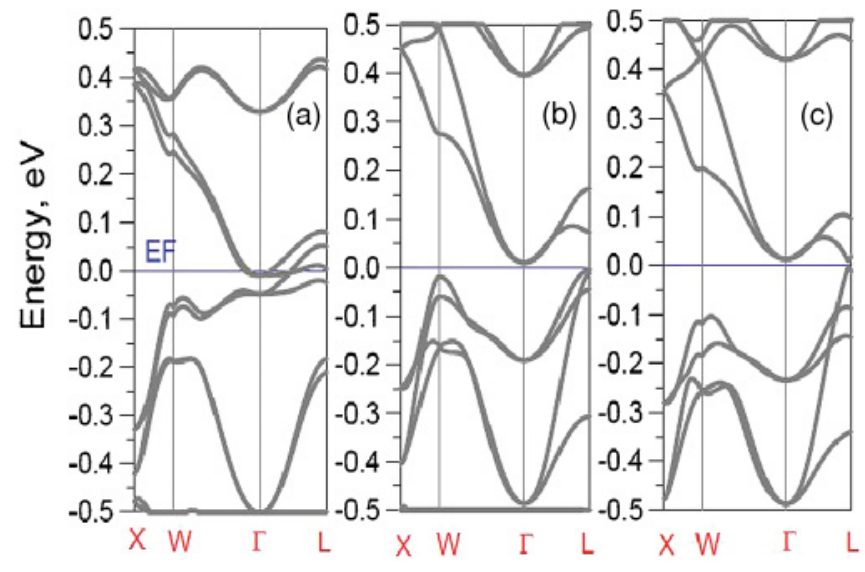

FIG. 3. (Color online) Evolution of electronic band structure of $\mathrm{Y}_{2} \mathrm{Ir}_{2} \mathrm{O}_{7}$ shown along high-symmetry directions, calculated using the $\mathrm{LSDA}+U+\mathrm{SO}$ method with three different values of $U$ equal (a) 0 (metallic), (b) $1.5 \mathrm{eV}$, and (c) $2 \mathrm{eV}$ (insulator with small gap). The Weyl point that is present in case (b), is not visible along high-symmetry lines.

even a quite large $U(=5 \mathrm{eV})$ cannot open a band gap for the collinear configuration. On the other hand, we find that the electronic states for the noncollinear all-in/all-out magnetic state depend strongly on the actual value of $U$ used in the calculation. In particular, we predict that when $U$ is less than $1 \mathrm{eV}$, the ground state is a normal metal while if $U$ is about $1.8 \mathrm{eV}$ or larger, we find the band structure to be insulating with an energy gap whose value depends on $U$.

Weak correlations. An interesting recent study proposed a tight-binding model for the nonmagnetic phase of the iridates, which was a topological insulator, ${ }^{8}$ a natural phase on the pyrochlore lattice. ${ }^{8,28}$ Our LDA studies of the realistic electronic structures contradict this; instead, we find a metallic phase [see Fig. 3(a)]. One can understand the discrepancy by analyzing the structure of energy levels at the $\Gamma$ point (Brillouin zone center) for the low-energy eight-band complex, composed of the four $J_{\text {eff }}=1 / 2$ states. In Ref. 8, these appear with degeneracies 4, 2, 2 (in order of increasing energy), which after filling with four electrons results in an insulating band structure. Our study of the nonmagnetic state using the LDA + SO method (with no $U$ ) results, on the other hand, in the sequence 2, 4, 2 of degeneracies, which is necessarily metallic assuming four levels are filled. A similar sequence of degeneracies was found in Ref. 19, where the simplified tight-binding model of Ref. 8 was extended to account for the nonoctahedral oxygen environment. It was shown that changing the geometry tunes one between the two degeneracy sequences. This effect is automatically built into our LDA calculations since the microscopic structure is used. According to Ref. 19, the noninsulating 2, 4, 2 sequence could ultimately lead to a topological insulator via a symmetry-breaking structural transition or uniaxial pressure.

Strong correlations and the Mott limit. When $U>1.8 \mathrm{eV}$, an insulating band structure is obtained with the all-in/all-out magnetic configuration, as shown in Fig. 3(c). Indeed, the band structure remains qualitatively similar on increasing $U$ to large values, where a site-localized moment is expected as in a Mott insulator. This can be further verified by calculating 

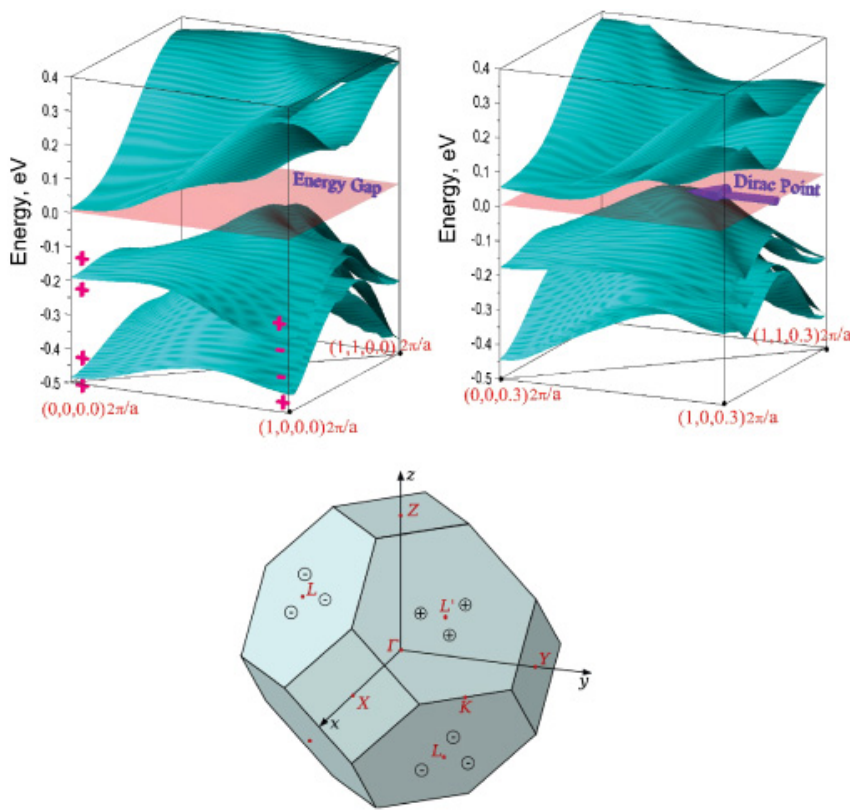

FIG. 4. (Color online) Semimetallic nature of the state at $U=$ $1.5 \mathrm{eV}$ according to the LSDA $+U+\mathrm{SO}$ method. (a) Calculated energy bands in the plane $K_{z}=0$ with band parities shown; (b) energy bands in the plane $k_{z}=0.6 \pi / a$, where a Weyl point is predicted to exist. The lighter-shaded plane is at the Fermi level. (c) Locations of the Weyl points in the three-dimensional Brillouin zone (Ref. 29) (nine are shown, indicated by the circled + or - signs).

the parity eigenvalues. Note that all the magnetic structures considered above preserve inversion (or parity) symmetry. In the Brillouin zone [see Fig. 4(c)] of the fcc lattice the TRIMs correspond to the $\Gamma=(0,0,0)$, and $X, Y, Z[=2 \pi / a(1,0,0)$ and permutations] points and four $L$ points $[\pi / a(1,1,1)$ and equivalent points]. The TRIM parities of the top four occupied bands, in order of increasing energy, are shown in Table II. Note that, although by symmetry all $L$ points are equivalent, the choice of inversion center at an iridium site singles out one of them, $L^{\prime}$. With that choice the parities at $L^{\prime}$ and the other three $L$ points are the opposite of one another. The parities of the all-in/all-out state remains unchanged above $U>U_{c} \sim$ $1.8 \mathrm{eV}$ and is shown in the top row under $U=2 \mathrm{eV}$. This pattern of parities helps to understand the nature of the phase: The parities are the same as for a site-localized picture of this phase, where each site has an electron with a fixed moment along the ordering direction. Due to the possibility of such a local description of this magnetic insulator, we term it the Mott phase.

Intermediate correlations. For the same all-in/all-out magnetic configuration, at smaller $U=1.5 \mathrm{eV}$, the band structure

TABLE II. Calculated parities of states at TRIMs for several electronic phases of the iridates. Only the top four filled levels are shown, in order of increasing energy.

\begin{tabular}{lcccc}
\hline \hline Phase & $\Gamma$ & $X, Y, Z$ & $L^{\prime}$ & $L(\times 3)$ \\
$U=2.0$, all-in (Mott) & ++++ & +--+ & +--- & -+++ \\
$U=1.5$, all-in (Dirac) & ++++ & +--+ & +--+ & -++- \\
\hline \hline
\end{tabular}

along high-symmetry lines [see Fig. 3(b)] also appears to be insulating, and at first sight one may conclude that this is an extension of the Mott insulator. However, a closer look using the parities reveals that a phase transition has occurred. At the $L$ points, an occupied level and an unoccupied level with opposite parities have switched places. It can readily be argued that only one of the two phases adjacent to the $U$ where this crossing happens can be insulating (see the Appendix). Since the large $U$ phase is found to be smoothly connected to a gapped Mott phase, it is reasonable to assume the smaller $U$ phase is the noninsulating one. This is also borne out by the LSDA $+U+\mathrm{SO}$ band structure. A detailed analysis perturbing about this transition point (also in the $\mathbf{k} \cdot \mathbf{p}$ subsection) allows us to show that this phase is expected to be a Weyl semimetal with 24 Weyl nodes in all.

Indeed, in the LSDA $+U+\mathrm{SO}$ band structure at $U=$ $1.5 \mathrm{eV}$, we find a three-dimensional Dirac crossing located within the $\Gamma-X-L$ plane of the Brillouin zone. This is illustrated in Fig. 4 and corresponds to the $\mathbf{k}$ vector $(0.52,0.52,0.3) 2 \pi / a$. There also are five additional Weyl points in the proximity of the point $L$ related by symmetry (three are just inside each of the two opposite hexagonal faces of the Brillouin zone, which are identified with one another) When $U$ increases, these points move toward each other and annihilate all together at the $L$ point close to $U=1.8 \mathrm{eV}$. This is how the Mott phase is born from the Weyl phase. Since we expect that for Ir $5 d$ states the actual value of the Coulomb repulsion should be somewhere within the range $1 \mathrm{eV}<U<2 \mathrm{eV}$, we thus conclude that the ground state of the $\mathrm{Y}_{2} \mathrm{Ir}_{2} \mathrm{O}_{7}$ is most likely the semimetallic state with the Fermi surface characterized by a set of Weyl points but in proximity to a Mott insulating state. Both phases can be switched to a normal metal if Ir moments are collinearly ordered by a magnetic field.

Possible axion insulator phase. At lower values of $U$ a second gapped phase with special properties may appear. This phase can be characterized in terms of its magnetoelectric response. Recall that in the presence of time-reversal symmetry, topological insulators are nonmagnetic band insulators with protected surface states. ${ }^{6}$ When the surface states are eliminated by adding, for example, magnetic moments only on the surface, a quantized magnetoelectric response is obtained: ${ }^{13}$ A magnetic field induces a polarization, $\mathbf{P}=\theta \frac{e^{2}}{2 \pi h} \mathbf{B}$, with the coefficient $\theta$ only defined modulo $2 \pi$. The values of $\theta$ are limited by time reversal, which transforms $\theta \rightarrow-\theta$. Apart from the trivial solution $\theta=0$, the ambiguity in the definition of $\theta$ allows also for $\theta=\pi$, and this occurs in topological insulators $\theta=\pi$. In magnetic insulators, $\theta$ is in general no longer quantized. ${ }^{30}$ However, when inversion symmetry is retained, $\theta$ is quantized again. An insulator with the value $\theta=\pi$ may be termed an axion insulator.

What is the appropriate description of the pyrochlore iridates? As described elsewhere, ${ }^{21}$ the condition for $\theta=\pi$ insulators with only inversion symmetry, when deduced from the parities, turns out to be the same as the Fu-Kane formula, for time-reversal symmetric insulators, ${ }^{31,32}$ that is, if the total number of filled states of negative parity at all TRIMs taken together is twice an odd integer, then $\theta=\pi$. Otherwise, $\theta=0$.

For the Mott insulator, at large $U$, the charge physics must be trivial and so we must have $\theta=0$. Next, since the Weyl 

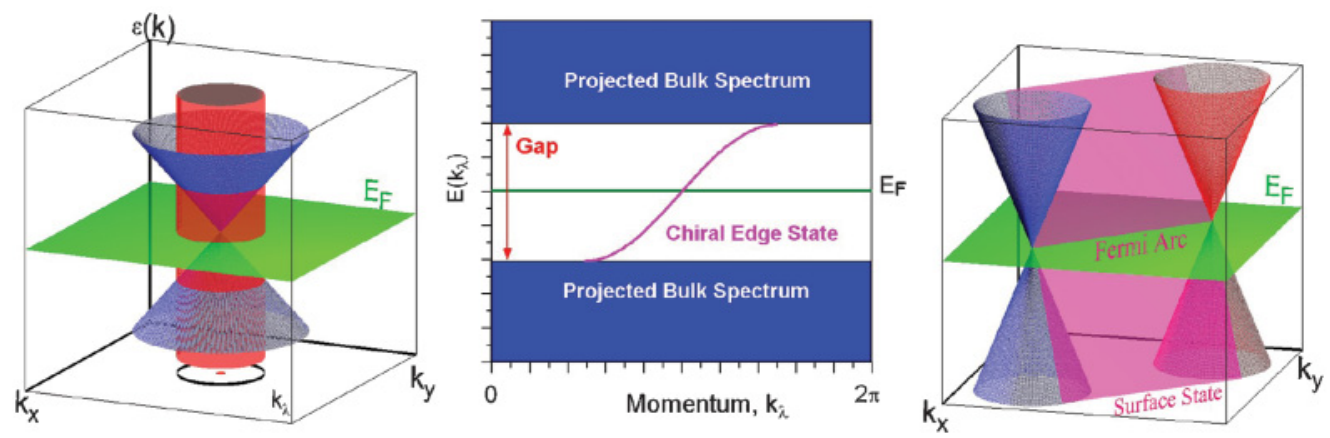

FIG. 5. (Color online) Illustration of surface states arising from bulk Weyl points. (a) The bulk states as a function of $\left(k_{x}, k_{y}\right)$ (and arbitrary $k_{z}$ ) fill the inside of a cone. A cylinder whose base defines a one-dimensional circular Brillouin zone is also drawn. (b) The cylinder unrolled onto a plane gives the spectrum of the two-dimensional subsystem $H\left(\lambda, k_{z}\right)$ with a boundary. On top of the bulk spectrum, a chiral state appears due to the nonzero Chern number. (c) Meaning of the surface states back in the three-dimensional system. The chiral state appears as a surface connecting the original Dirac cone to a second one, and the intersection between this plane and the Fermi level gives a Fermi arc connecting the Weyl points.

semimetal phase is gapless in the bulk, $\theta$ is ill defined. As $U$ is lowered further, the Weyl points shift, with nodes of opposite chirality approaching one another. If at lower values of $U$ the Weyl points meet and annihilate again, the resulting phase will have $\theta=\pi$. The parities will be the same as in the Weyl semimetal, since the Weyl points would have annihilated away from TRIMs. From Table II we can see that indeed this corresponds to $\theta=\pi$, since there are 14 negative-parity filled states, while the Mott insulator corresponds to $\theta=0$, having 12 negative-parity filled states. Indeed, the presence of the intervening Weyl phase can be deduced from the requirement that $\theta$ has to change between these two quantized values. Similarly, when time-reversal symmetry is present but inversion absent, a gapless phase must intervene when a change in topology occurs. ${ }^{33}$

Unfortunately, within our LSDA $+U+\mathrm{SO}$ calculation, a metallic phase intervenes on lowering $U \leqslant 1.0 \mathrm{eV}$, before the Weyl points annihilate to give the axion insulator. We point out this possibility nevertheless, since LDA systematically underestimates the stability of such gapped phases. Moreover, it provides an interesting example of a pair of insulators, a Mott insulator and a smaller $U$ "Slater" insulator, with the same magnetic order, but which are nevertheless different phases, which cannot be smoothly connected to one another. Inversion symmetry is critical in preserving this distinction.

Topological Weyl semimetal. In the semimetal phase, there are 24 Weyl points. Near each $L$ point there are 3 Weyl points related by the threefold rotation, which have the same chiral charge, as well as the inverse images with opposite chirality. Thus, there are 24 Weyl points, where the valence and conduction band line up, in the whole Brillouin zone. Since all are at the same energy by symmetry, the chemical potential must pass through them. The Fermi velocities at the Weyl point are found to be typically an order of magnitude smaller than in graphene. We briefly note that this Weyl semimetal is a gapless state with power-law forms for various properties, which will be described in more detail elsewhere. For example, the density of states $N(E) \propto E^{2}$. The small density of states makes this an insulator at zero temperature and frequency (as seen experimentally for $\mathrm{Y}_{2} \mathrm{Ir}_{2} \mathrm{O}_{7}$ ). The ac conductivity for a single node with isotropic velocity $v$ in the free particle limit of the clean system is $\sigma(\Omega)=$ $\frac{e^{2}}{12 h} \frac{|\Omega|}{v}$. Furthermore, novel magnetoconductance phenomena are expected in Weyl semimetals, as a consequence of the Adler-Bell-Jackiw anomaly of Weyl fermions, ${ }^{34}$ which will be discussed in future work. Additionally, a Weyl metal must automatically have surface states, as explained in the next section.

\section{SURFACE STATES}

The Weyl points behave like "magnetic" monopoles in momentum space whose charge is given by the chirality; they are actually a source of "Berry flux" rather than magnetic flux. The Berry connection, a vector potential in momentum space, is defined by $\mathcal{A}(\mathbf{k})=\sum_{n=1}^{N} i\left\langle u_{n \mathbf{k}}\left|\nabla_{\mathbf{k}}\right| u_{n \mathbf{k}}\right\rangle$, where $N$ is the number of occupied bands. As usual, the Berry flux is defined as $\mathcal{F}=\nabla_{\mathbf{k}} \times \mathcal{A}$. To show that there are arcs connecting pairs of Weyl points, we argue that there is an arc on the surface Brillouin zone emanating from the projection $\left(k_{0 x}, k_{0 y}\right)$ of each Weyl point.

The argument is based on the band topology around the Weyl points. Consider a curve in the surface Brillouin zone encircling the projection of the bulk Weyl point, which is traversed counterclockwise as we vary the parameter $\lambda: 0 \rightarrow 2 \pi$; $\mathbf{k}_{\lambda}=\left[k_{x}(\lambda), k_{y}(\lambda)\right]$ [see Fig. 5(a)]. We show that the energy $\epsilon_{\lambda}$ of a surface state at momentum $\mathbf{k}_{\lambda}$ crosses $E=0$ at some value of $\lambda$. Consider $H\left(\lambda, k_{z}\right)=H\left(\mathbf{k}_{\lambda}, k_{z}\right)$, which can be interpreted as the gapped Hamiltonian of a two-dimensional system (with $\lambda$ and $k_{z}$ as the two momenta). The two periodic parameters $\lambda, k_{z}$ define the surface of a torus in momentum space. The Chern number of this two-dimensional band structure is given by the Berry curvature integration: $\frac{1}{2 \pi} \int \mathcal{F} d k_{z} d \lambda$, which, by the Stokes theorem, simply corresponds to the net monopole density enclosed within the torus. This is obtained by summing the chiralities of the enclosed Weyl nodes. Consider the case when the net chirality is unity, corresponding to a single enclosed Dirac node. Then, the two-dimensional subsystem is a quantum Hall insulator with unit Chern number. When the system is given a boundary at $z=0$, we expect a chiral edge 
state for this subsystem [see Fig. 5(b)]. Hence, this surface state crosses zero energy somewhere on the surface Brillouin zone $\mathbf{k}_{\lambda_{0}}$. Such a state can be obtained for every curve enclosing the Weyl point. Thus, at zero energy, there is a Fermi line in the surface Brillouin zone, that terminates at the Weyl point momenta [see Fig. 5(c)]. An arc beginning on a Weyl point of chirality $c$ has to terminate on a Weyl point of the opposite chirality. Clearly, the net chirality of the Weyl points within the $\left(\lambda, k_{z}\right)$ torus was a key input in determining the number of these states. If Weyl points of opposite chirality line up along the $k_{z}$ direction, then there is a cancellation and no surface states are expected.

In the calculations for $\mathrm{Y}_{2} \mathrm{Ir}_{2} \mathrm{O}_{7}$, at $U=1.5 \mathrm{eV}$, a Dirac (or Weyl) node is found to occur at the momentum $(0.52,0.52,0.30) 2 \pi / a$ (in the coordinate system aligned with the cubic lattice of the crystal) and equivalent points (see Fig. 4). They can be thought of as occurring on the edges of a cube, with a pair of Dirac nodes of opposite chirality occupying each edge, as, for example, the points $(0.52,0.52,0.30) 2 \pi / a$ and $(0.52,0.52,-0.30) 2 \pi / a$. For the case of $U=1.5 \mathrm{eV}$, the sides of this cube have the length $0.52(4 \pi / a)$. Thus, the (111) and (110) surfaces would have surface states connecting the projected Weyl points [see Fig. 6 for the (110) surface states and the theoretical expectation for the (111) surface]. If, on the other hand, we consider the surface orthogonal to the (001) direction, Weyl points of opposite chirality are projected to the same surface momentum along the edges of the cube. Thus, no protected states are expected for this surface.

To verify these theoretical considerations, we have constructed a tight-binding model which has features seen in our electronic structure calculations for $\mathrm{Y}_{2} \mathrm{Ir}_{2} \mathrm{O}_{7}$. The calculated (110) surface band structure for the slab of 128 atoms together with the sketch of the obtained Fermi arcs is shown in Fig. 6. This figure shows Fermi arcs from both the front and the back face of the slab, so there are twice as many arcs coming out of each Weyl point as predicted for a single surface.

The tight-binding model considers only $t_{2 g}$ orbitals of Ir atoms in the global coordinate system. Since Ir atoms form a tetrahedral network (see Fig. 2), each pair of nearestneighboring atoms forms a corresponding $\sigma$-like bond whose hopping integral is denoted as $t$ and another two $\pi$-like bonds whose hopping integrals are denoted as $t^{\prime}$. To simulate the appearance of the Weyl point it is essential to include next-nearest-neighbor interactions between $t_{2 g}$ orbitals which are denoted as $t^{\prime \prime}$. With the parameters $t=0.2, t^{\prime}=$ $0.5 t, t^{\prime \prime}=-0.2 t$, the value of the on-site spin-orbit coupling equal to $2.5 t$ and the applied on-site "Zeeman" splitting of $0.1 t$ between states parallel and antiparallel to the local quantization axis of the all-in/all-out configuration we can roughly model the bulk Weyl semimetal state; when this model is solved on a lattice with a boundary, the surface states shown in the figure appear.

\section{DISCUSSION}

We now discuss how the present theoretical description compares with experimental facts. We propose that the lowtemperature state of $\mathrm{Y}_{2} \mathrm{Ir}_{2} \mathrm{O}_{7}$ (and also possibly of $A=$ $\mathrm{Eu}, \mathrm{Sm}$, and $\mathrm{Nd}$ iridates) is a Weyl semimetal, with allin/all-out magnetic order. This is broadly consistent with the

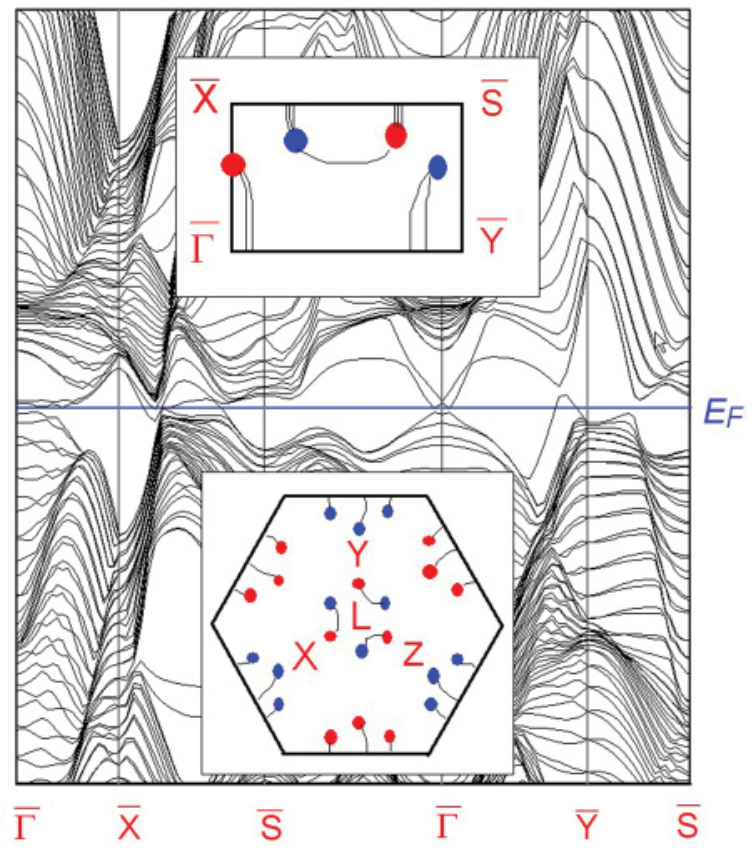

FIG. 6. (Color online) Surface states. The calculated surface energy bands correspond to the (110) surface of the pyrochlore iridate $\mathrm{Y}_{2} \mathrm{Ir}_{2} \mathrm{O}_{7}$. A tight-binding approximation has been used to simulate the bulk band structure with three-dimensional Weyl points as found by our LSDA $+U+\mathrm{SO}$ calculation. The plot corresponds to diagonalizing 128 atoms slab with two surfaces. The upper inset shows a sketch of the deduced Fermi arcs connecting projected bulk Weyl points of opposite chirality. The inset below sketches the theoretically expected surface states on the (111) surface at the Fermi energy (surface band structure not shown for this case).

interconnection between insulating behavior and magnetism observed experimentally. ${ }^{9,10}$ It is also consistent with being proximate to a metallic phase on lowering the correlation strength, such as $A=\operatorname{Pr}$ (Ref. 17). In the clean limit, a threedimensional Weyl semimetal is an electrical insulator and can potentially account for the observed electrical resistivity. The noncollinear magnetic order proposed has Ising symmetry and could undergo a continuous ordering transition. The observed "spin-glass"-like magnetic signature could perhaps arise from defects like magnetic domain walls. A direct probe of magnetism is currently lacking and would shed light on this key question. At lower values of $U$, the system may realize an "axion insulator" phase with a magnetoelectric response $\theta=\pi$, although within our calculations (which are known to underestimate stability of such gapped phases) a Fermi surface appears before this happens.

In summary, a theoretical phase diagram for the physical system is shown in Fig. 1 as a function of $U$ and applied magnetic field, which leads to a metallic state beyond a critical field. The precise nature of these phase transformations is not addressed in the present study.

Note: An experimental paper ${ }^{35}$ appeared recently in which it is found that the spins in a related compound $\left(\mathrm{Eu}_{2} \mathrm{Ir}_{2} \mathrm{O}_{7}\right)$ form a regularly ordered state rather than a spin-glass, consistent with our results. It would be interesting to learn whether this compound is a Weyl metal or not. 


\section{ACKNOWLEDGMENTS}

A.V. thanks L. Balents, J. Orenstein, and R. Ramesh for insightful discussions. X.W. acknowledges support by National Key Project for Basic Research of China (Grants No. 2011CB922101 and No. 2010CB923404) and NSFC under Grants No. 10774067 and No. 10974082. S.S. acknowledges support by DOE SciDAC Grant No. SE-FC02-06ER25793 and thanks Nanjing University for the kind hospitality during his visit to China. X.W. and S.S also acknowledge support from Kavli Institute for Theoretical Physics where this work was initiated. We also acknowledge support by the Laboratory Directed Research and Development Program of Lawrence Berkeley National Laboratory under US Department of Energy Contract No. DE-AC02-05CH11231 (A.M.T.) as well as support by the National Science Foundation under grant NSF-DMR 0645691 (A.V.).

\section{APPENDIX: EFFECTIVE $k \cdot p$ THEORY AND INTERVENING WEYL SEMIMETAL PHASE}

We have been using inversion symmetry which allows us to track wave-function parities of occupied states at TRIMs. Near electronic phase transitions where these parities change, a low-energy $\mathbf{k} \cdot \mathbf{p}$ theory helps to understand qualitative features of the neighboring phases. Consider a pair of states at the $L$ point which have opposite parity and cross each other as we tune $U$. We want to understand what happens to the band structure.

The $L$ point has three symmetries which do not change its crystal momentum. First, of course, is inversion, and we can label states by the eigenvalues $P= \pm 1$. The second is $120^{\circ}$ rotations about a line joining $L-\Gamma$. There are three possible eigenvalues which we call $s=-1 / 2,1 / 2,3 / 2$. So any state at this point can be labeled by $\{P, s\}$. The third symmetry is described below. Now consider writing the effective $2 \times 2$ Hamiltonians for the pair of states just above and below the Fermi energy:

(i) At the $L$ point. Since we have inversion symmetry and the two states have distinct eigenvalues $P= \pm 1$, they cannot mix. Hence, the effective Hamiltonian is

$$
H(L)=\Delta \sigma_{z},
$$

where the coefficient $\Delta$ changes sign when the levels pass through each other. (Parity is represented by $\sigma_{z}$.) For the system in question, $\Delta$ changes from positive to negative as $U$ increases (below the transition, the occupied state is odd according to Table I). Note that the $s$ quantum number of the two levels is irrelevant here.

(ii) Along the $\Gamma-L$ direction. We still have the quantum number $s$, but not $P$, since inversion changes the momentum. Denoting by $q_{z}$ the deviation of the momentum along this line from the $L$ point, we have two cases. If the $s$ quantum number of the two levels is different, they still cannot mix, so the effective Hamiltonian is $H=\left(\Delta+\frac{q_{z}^{2}}{2 m_{1}}\right) \sigma_{z}$. Now, when $m_{1} \Delta<0$ there are two values $q_{z}= \pm \sqrt{-2 m_{1} \Delta}$, where there are nodes along this $\Gamma-L$ line. One can see such crossings between other pairs of modes, which must have different spins, in the data [Figs. 3(b) and 3(c)]. However, if the states have the same $s$ quantum number they can mix, once you move away from $L$. This is the case for the levels that switch places in the transition we are interested in, the one near $U=1.8 \mathrm{eV}$ in our study of $\mathrm{Y}_{2} \mathrm{Ir}_{2} \mathrm{O}_{7}$. Now the effective Hamiltonian could be

$$
H(\Gamma-L)=\left(\Delta+\frac{q_{z}^{2}}{2 m_{1}}\right) \sigma_{z}+\beta q_{z} \sigma_{y} .
$$

Now the spectrum is $E=\sqrt{\left(q_{z}^{2}-|\Delta|\right)^{2}+q_{z}^{2}}$, for $\Delta<0$, so despite a level crossing there is no node along the $\Gamma$ - $L$ line for any value of $\Delta$.

(iii) General point in BZ. In the latter case, does this mean there are no Dirac points? No, we just need to move off the $\Gamma$ - $L$ line. Let the deviation be $\mathbf{q}_{\perp}$, a 2 vector. The fact that the two levels have opposite parity means we need an odd function of $\mathbf{q}_{\perp}$ to induce a matrix element between the levels. Also, the rotation transforms $\mathbf{q}_{\perp}$ as a vector but does not affect $\sigma_{x}$ and $\sigma_{y}$ (the two-component wave function is not an ordinary spinor since both components have the same "spin" s). Consequently, only the combinations $q_{\perp}^{3} \cos 3 \theta$ and $q_{\perp}^{3} \sin 3 \theta$, which are invariant under the rotation, can appear in the Hamiltonian. Here $\theta$ is the angle between $\mathbf{q}_{\perp}$ and the plane containing $\Gamma-L-K$ (see Fig. 4); thus, the allowed form for the $q_{\perp}$ coupling is $\Delta H=c_{1} q_{\perp}^{3} \cos 3 \theta \sigma_{y}+c_{2} q_{\perp}^{3} \sin 3 \theta \sigma_{x}$. (Note that the coupling of the $\cos$ and $\sin$ to $\sigma_{y}$ and $\sigma_{x}$, respectively, is dictated by a third symmetry of the all-in/all-out magnetic order: namely, reflection in the $\Gamma-L-K$ plane followed by time reversal. We adjust the phases so that both states have an eigenvalue of +1 for this antiunitary transformation.) Putting this all together with an additional effective mass term $\frac{q_{\perp}^{2}}{2 m_{2}} \sigma_{z}$, we have the effective Dirac Hamiltonian near the $L$ point:

$$
\begin{aligned}
H(\mathbf{q})= & \left(\Delta+\frac{q_{z}^{2}}{2 m_{1}}+\frac{q_{\perp}^{2}}{2 m_{2}}\right) \sigma_{z}+\left(\beta q_{z}+c_{1} q_{\perp}^{3} \cos 3 \theta\right) \sigma_{y} \\
& +c_{2} q_{\perp}^{3} \sin 3 \theta \sigma_{x} .
\end{aligned}
$$

Note that this has the form $A(q) \sigma_{z}+B(q) \sigma_{y}+C(q) \sigma_{x}$. For a node, $A=B=C=0$. According to the electronic structure calculations for $\mathrm{Y}_{2} \mathrm{Ir}_{2} \mathrm{O}_{7}, m_{2}<0$ and $m_{1}>0$. The Dirac nodes then are determined by (i) $C=0 \rightarrow \theta=p \pi / 3$ and (ii) using this, $B=0 \rightarrow q_{z}= \pm c_{1} q_{\perp}^{3} / \beta$, depending on whether we look at $p=$ odd or $p=$ even. Finally, using these relations we have for the $A=0$ equation:

$$
\Delta+c_{1}^{2} \frac{q_{\perp}^{6}}{2 \beta^{2} m_{1}}-\frac{q_{\perp}^{2}}{2\left|m_{2}\right|}=0
$$

For small $\Delta$ there is a small solution; thus, $q_{\perp}^{2} \approx 2\left|m_{2}\right| \Delta$. Note that this has a solution only for $\Delta>0$, that is, before the gap gets inverted at the $L$ point on increasing $U$. Thus, in this scenario, there is a Weyl point only in the small $U$ phase. These Weyl points are arranged as in Fig. 4. If $m_{2}$ is positive, there is a transition from the $\theta=\pi$ magnetic axion insulator to the Weyl semimetal on increasing $U$, contradicting its evolution into a Mott insulator. 
${ }^{1}$ J. Orenstein and A. J. Millis, Science 288, 468 (2000).

${ }^{2}$ Y. Tokura and N. Nagaosa, Science 288, 462 (2000)

${ }^{3}$ B. J. Kim, Hosub Jin, S. J. Moon, J.-Y. Kim, B.-G. Park, C. S. Leem, Jaejun Yu, T. W. Noh, C. Kim, S.-J. Oh, J.-H. Park, V. Durairaj, G. Cao, and E. Rotenberg, Phys. Rev. Lett. 101, 076402 (2008); B. J. Kim, H. Ohsumi, T. Komesu, S. Sakai, T. Morita, H. Takagi, and T. Arima, Science 323, 1329 (2009).

${ }^{4}$ Y. Okamoto, M. Nohara, H. Aruga-Katori, and H. Takagi, Phys. Rev. Lett. 99, 137207 (2007).

${ }^{5}$ P. A. Lee, Science 321, 1306 (2008); L. Balents, Nature (London) 464, 199 (2010).

${ }^{6}$ M. Z. Hasan and C. L. Kane, Rev. Mod. Phys. 82, 3045 (2010); J. E. Moore, Nature (London) 464, 194 (2010); X. L. Qi and S. C. Zhang, Phys. Today 63, 33 (2010).

${ }^{7}$ A. Shitade, H. Katsura, J. Kunes̃, X. L. Qi, S. C. Zhang, and N. Nagaosa, Phys. Rev. Lett. 102, 256403 (2009).

${ }^{8}$ D. A. Pesin and L. Balents, Nat. Phys. 6, 376 (2010).

${ }^{9}$ N. Taira, M. Wakeshima, and Y. Hinatsu, J. Phys. Condens. Matter 13, 5527 (2001).

${ }^{10}$ K. Matsuhira, M. Wakeshima, R. Nakanishi, T. Yamada, A. Nakamura, W. Kawano, S. Takagi, and Y. Hinatsu, J. Phys. Soc. Jpn. 76, 043706 (2007).

${ }^{11}$ A. K. Geim and K. S. Novoselov, Nat. Mater. 6, 183 (2007).

${ }^{12}$ H. B. Nielsen and M. Ninomiya, Nucl. Phys. B 193, 173 (1981).

${ }^{13}$ X. L. Qi., T. L. Hughes, and S. C. Zhang, Phys. Rev. B 78, 195424 (2008).

${ }^{14}$ F. Wilczek, Phys. Rev. Lett. 58, 1799 (1987).

${ }^{15}$ M. A. Subramanian, G. Aravamudan, and G. V. Subba Rao, Prog. Solid St. Chem. 15, 55 (1983); S. T. Bramwell and M. J. P. Gingras, Science 294, 1495 (2001); A. P. Ramirez, Ann. Rev. Mater. Sci. 24, 453 (1994).

${ }^{16}$ J. S. Gardner, M. J. P. Gingras, and J. E. Greedan, Rev. Mod. Phys. 82, 53 (2010).

${ }^{17}$ D. Yanagishima and Y. Maeno, J. Phys. Soc. Jpn. 70, 2880 (2001).
${ }^{18}$ K. Maiti, Solid State Commun. 149, 1351 (2009).

${ }^{19}$ B. J. Yang and Y. B. Kim, Phys. Rev. B 82, 085111 (2010).

${ }^{20}$ Systems with time-reversal but not inversion symmetry can have Weyl points. However, in this case both $\pm \mathbf{k}_{\mathbf{0}}$-centered Weyl points have the same chirality. Weyl points of opposite chirality (of which there must be an equal number) are then typically not related by symmetry, so the Weyl points might not be at the Fermi energy.

${ }^{21}$ Ari M. Turner, Yi Zhang, Roger S. K. Mong, and Ashvin Vishwanath, e-print arXiv:1010.4335 (to be published); T. L. Hughes, E. Prodan, and A. B. Bernevig, e-print arXiv:1010.4508 (to be published).

${ }^{22}$ S. Y. Savrasov, Phys. Rev. B 54, 16470 (1996).

${ }^{23}$ V. I. Anisimov, F. Aryasetiawan, and A. I. Lichtenstein, J. Phys. Condens. Matter 9, 767 (1997).

${ }^{24}$ R. Siddharthan, B. S. Shastry, A. P. Ramirez, A. Hayashi, R. J. Cava, and S. Rosenkranz, Phys. Rev. Lett. 83, 1854 (1999); M. J. Harris, S. T. Bramwell, D. F. McMorrow, T. Zeiske, and K. W. Godfrey, ibid. 79, 2554 (1997).

${ }^{25}$ M. Elhajal, B. Canals, R. Sunyer, and C. Lacroix, Phys. Rev. B 71, 094420 (2005).

${ }^{26}$ H. Jin, H. Kim, H. Jeong, C. H. Kim, and J. Yu, e-print arXiv:0907.0743 (to be published).

${ }^{27}$ Y. Singh and P. Gegenwart, Phys. Rev. B 82, 064412 (2010).

${ }^{28}$ H. M. Guo and M. Franz, Phys. Rev. Lett 103, 206805 (2009).

${ }^{29}$ N. W. Ashcroft and N. D. Mermin, Solid State Physics (Saunders, Philadelphia, 1976).

${ }^{30}$ A. Malashevich, I. Souzo, S. Coh, and D. Vanderbilt, New J. Phys. 12, 053032 (2010); A. M. Essin, A. M. Turner, J. E. Moore, and D. Vanderbilt, Phys. Rev. B 81, 205104 (2010).

${ }^{31}$ L. Fu and C. L. Kane, Phys. Rev. B 76, 045302 (2007).

${ }^{32}$ Z. Wang, X. L. Qi, and S. C. Zhang, New J. Phys. 12, 065007 (2010).

${ }^{33}$ Shuichi Murakami, New J. Phys. 9, 356 (2007).

${ }^{34}$ H. B. Nielsen and Masao Ninomiya, Phys. Lett. B 130, 389 (1983)

${ }^{35}$ Zhao et al., e-print arXiv:1104.1213 (unpublished). 